\title{
Simulating Wilson fermions without critical slowing down
}

\author{
Urs Wenger* \\ Albert Einstein Center for Fundamental Physics \\ Institute for Theoretical Physics \\ University of Bern, Sidlerstr. 5, CH-3012 Bern, Switzerland \\ E-mail: wengereitp.unibe.ch
}

We present a simulation algorithm for Wilson fermions based on the exact hopping expansion of the fermion action. The algorithm essentially eliminates critical slowing down by sampling the fermionic two-point correlation function and it allows simulations directly in the massless limit. As illustrative examples, the algorithm is applied to the Gross-Neveu and the Schwinger model, the latter in the strong coupling limit.

The XXVII International Symposium on Lattice Field Theory - LAT2009

July 26-31 2009

Peking University, Beijing, China

${ }^{*}$ Speaker. 


\section{Introduction}

Simulating strongly interacting fermions continues to be a major challenge in computational physics. The standard procedure to deal with fermionic degrees of freedom is to integrate out the fermionic fields in order to obtain the fermion determinant det $D$, where $D$ denotes the Dirac operator. However, this procedure is not unproblematic. Consider for example a fermion interacting with a bosonic field $U$. After integrating out the fermion fields one obtains det $D(U)$ which yields an effective action non-local in the bosonic field. The standard method is now to re-express the determinant using bosonic 'pseudo-fermions' and use the Hybrid Monte Carlo algorithm [1] which in essence encodes the non-locality of the fermion determinant in the inverse $D(U)^{-1}$. Another problem is that the standard approach suffers from critical slowing down (CSD) towards the chiral limit. In that limit the correlation length of the fermionic two-point function diverges. As a consequence the Dirac operator $D(U)$ develops very small modes and eventually the inverse $D(U)^{-1}$ becomes ill-conditioned. Yet another problem concerns the phase of $\operatorname{det} D$ which for Wilson fermions is in general non-zero. Hence a probabilistic interpretation of the integration measure, necessary for any Monte Carlo simulation, is not possible and leads to a sign problem when an odd number of Wilson fermion flavours is simulated.

Here we propose a novel approach [2] circumventing the above mentioned problems. It is based on the exact hopping expansion of the fermion action, i.e. a reformulation of the fermion system as a statistical closed loop model. We develop a simulation algorithm which samples directly the fermionic two-point function and in this way eliminates CSD. Moreover, it allows to specify the fermionic boundary conditions a posteriori, i.e. after the simulation, and allows simulations directly in the massless limit. The approach is applicable to the Gross-Neveu (GN) model in $D=2$ dimensions, to the Schwinger model in the strong coupling limit in $D=2$ and $D=3$ dimensions, to supersymmetric quantum mechanics and the $N=1$ and 2 supersymmetric Wess-Zumino model in $D=2$ dimensions. In the present proceedings we concentrate on the application to the GN and the Schwinger model.

Finally, we would like to emphasise that the reformulation based on the hopping expansion is not new $[3,4,5,6]$. Mostly, however, it has been applied to staggered fermions in the strong coupling limit where a reformulation in terms of monomers and dimers [7] allows efficient algorithms $[8,9]$ that were subsequently applied to many interesting systems $[10,11,12]$, see also the recent review by Chandrasekharan [13]. For Wilson fermions on the other hand the loop formulation has been developed for the Schwinger model in the strong coupling limit [14] and the GN model $[5,6,15]$ and what we propose in [2] is just a very efficient algorithm for these loop formulations.

\section{Loop formulation of Wilson fermions}

We start with the reformulation of $D=2$ fermionic systems involving Wilson fermions in terms of a statistical loop gas model. We use the GN model, a prototype for strongly interacting fermions, as an illustrative example. The model is most naturally formulated in terms of Majorana fermions. Employing the Wilson lattice discretisation for a Majorana fermion the Euclidean Lagrange density reads

$$
\mathscr{L}=\frac{1}{2} \xi^{T} \mathscr{C}\left(\gamma_{\mu} \tilde{\partial}_{\mu}-\frac{1}{2} \partial^{*} \partial+m\right) \xi-\frac{g^{2}}{4}\left(\xi^{T} \mathscr{C} \xi\right)^{2}
$$


where $\xi$ is a real 2-component Grassmann field, $\mathscr{C}=-\mathscr{C}^{T}$ is the charge conjugation matrix and $\partial, \partial^{*}$ and $\tilde{\partial}$ are the forward, backward and the symmetric lattice derivative, respectively. In the continuum, the massless model enjoys a discrete chiral symmetry $\xi \rightarrow \gamma_{5} \xi$ which on the lattice is broken explicitly by the Wilson term $\frac{1}{2} \partial^{*} \partial$. The symmetry can be restored in the continuum by fine tuning $m \rightarrow m_{c}$. Further we note that a pair of Majorana fermions may be considered as one Dirac fermion, i.e. $\psi=1 / \sqrt{2}\left(\xi_{1}+i \xi_{2}\right), \quad \bar{\psi}=1 / \sqrt{2}\left(\xi_{1}^{T}-i \xi_{2}^{T}\right) \mathscr{C}$, exposing the $O(2 N)$ flavour symmetry explicitly. Since integrating out Majorana fermions yields the Pfaffian of the antisymmetric Dirac operator, the model with $2 N$ Majorana fermions is equivalent to $N$ Dirac fermions through the identity $(\operatorname{Pf} D)^{2 N}=(\operatorname{det} D)^{N}$.

At non-vanishing coupling $g \neq 0$ one usually employs a Hubbard-Stratonovich transformation and introduces the scalar field $\sigma \propto \xi^{T} \mathscr{C} \xi$. With $M(x)=2+m+\sigma(x)$ and $P( \pm \mu)=\frac{1}{2}\left(1 \mp \gamma_{\mu}\right)$ the action then becomes the sum of monomer and hopping terms

$$
S_{\mathrm{GN}}=\frac{1}{2} \sum_{x} \xi^{T}(x) \mathscr{C} M(x) \xi(x)-\sum_{x, \mu} \xi^{T}(x) \mathscr{C} P(\mu) \xi(x+\hat{\mu}) .
$$

Using the nil-potency of Grassmann elements one can now expand the Boltzmann factor and perform an exact hopping expansion for the Majorana Wilson fermions [15]. We emphasise that this can be done for any fermionic theory (bilinear in the fermionic fields). At each site, the fields $\xi^{T} \mathscr{C}$ and $\xi$ must be exactly paired in order to give a non-vanishing contribution to the path integral,

$$
\int \mathscr{D} \xi \prod_{x}\left(M(x) / 2 \xi^{T}(x) \mathscr{C} \xi(x)\right)^{m(x)} \prod_{x, \mu}\left(\xi^{T}(x) \mathscr{C} P(\mu) \xi(x+\hat{\mu})\right)^{b_{\mu}(x)}
$$

where the occupation numbers $m(x)=0,1$ for monomers and $b_{\mu}(x)=0,1$ for bonds (or dimers) satisfy the constraint

$$
m(x)+\frac{1}{2} \sum_{\mu} b_{\mu}(x)=1 .
$$

This constraint encodes that only closed, non-intersecting paths survive the integration and we end up with a closed loop representation of the partition function in terms of monomers and dimers, i.e. $Z=\sum_{\ell} \omega(\ell)$. The weight $\omega$ of each loop $\ell$ can be calculated analytically $[5,6,15,16]$ yielding $|\omega(\ell)|=2^{-c / 2}$ where $c$ is the number of corners in the loop, while the phase of $\omega(\ell)$ depends on the geometrical shape of $\ell$. In $D=2$ dimensions and for a torus geometry of the lattice, $\operatorname{sign}[\omega(\ell)] \in\{-1,1\}$ depends on the boundary conditions (BC) $\varepsilon_{\mu} \in\{0,1\}$ and on the number $n_{\mu}$ of loop windings in direction $\mu$,

$$
\operatorname{sign}[\omega(\ell)]=(-1)^{n_{\mu}\left(\varepsilon_{\mu}+n_{\mu}\right)} .
$$

As a consequence the overall sign of a given configuration depends only on the fermionic BC and the total winding number $l=\left\{l_{\mu}\right\}$ (modulo 2).

If we separate all configurations into the equivalence classes $\mathscr{L}_{i j}$ where the subscripts $i, j$ specify the total winding numbers $l_{\mu}$ (modulo 2 ) in the two directions, then the partition function summing over all non-oriented, self-avoiding loops with positive weight,

$$
Z=\sum_{\{\ell\} \in \mathscr{L}}|\omega[\ell]| \prod_{x \notin \ell} M(x), \quad \mathscr{L} \in \mathscr{L}_{00} \cup \mathscr{L}_{10} \cup \mathscr{L}_{01} \cup \mathscr{L}_{11}
$$



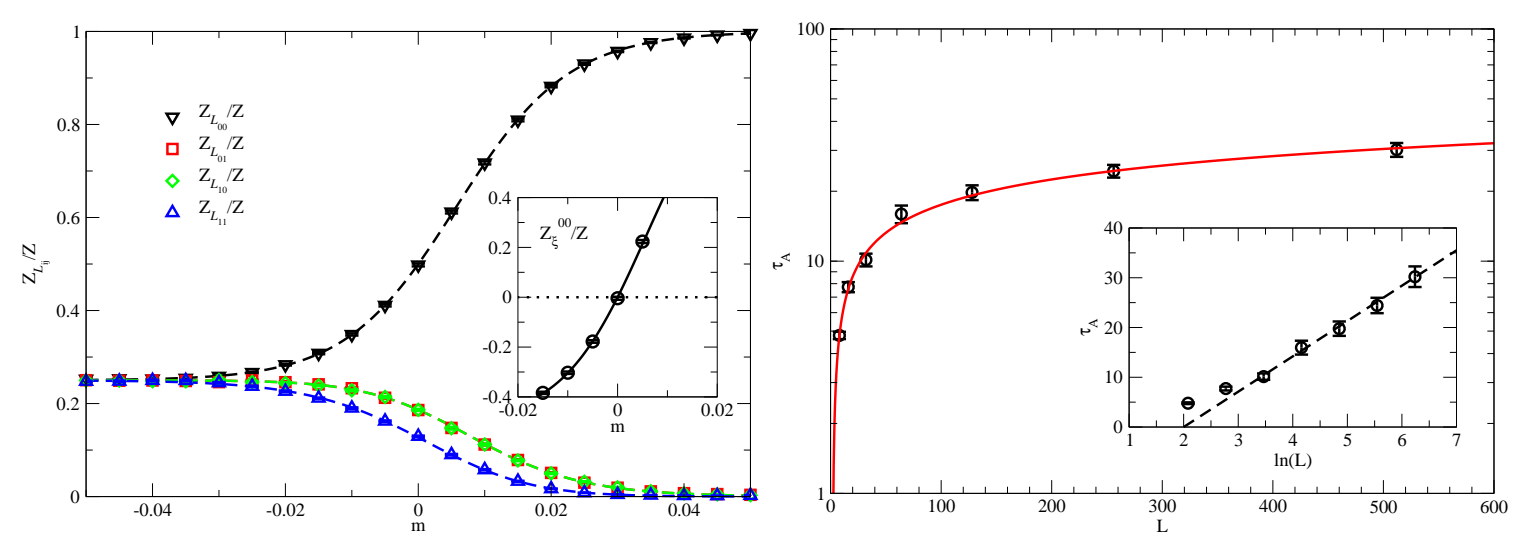

Figure 1: $N=1$ Majorana GN model on a $128^{2}$ lattice. Left: Comparison of simulation results (symbols) and analytic calculations (dashed lines) for the partition function ratios $Z_{\mathscr{L}_{i j}} / Z$. The inset shows the reproduction of the zero mode of $Z_{\xi}^{00}$ at $m_{c}=0$. Right: Integrated autocorrelation time of the condensate at the critical point $m_{c}=0$ fitted by $\tau_{A} \sim L^{z}$ with $z=0.31(4)$. The inset shows a fit to a logarithmic dependence on $L$.

represents a system with unspecified fermionic BC while systems with specific fermionic BC can be constructed a posteriori by taking the signs of each class according to

$$
Z_{\xi}^{\varepsilon}=2 Z_{\mathscr{L}_{00}}-\sum_{i, j=0}^{1}(-1)^{\varepsilon_{\mu} l_{\mu}} Z_{\mathscr{L}_{i j}} .
$$

Finally we note that if one considers $N>1$ Majorana flavours the occupation numbers $m, b_{\mu}$ are decorated by the flavour index $\alpha$ and one considers $N$ different loop flavours. The monomer weight $M(x)$ depends on the local fermion density $\sum_{\alpha} m^{\alpha}(x)$ only and one ends up with a model of locally coupled loops.

In the Schwinger model the hopping term contains a $U(1)$ phase coming from the gauge field $\phi_{\mu}(x)$, and the non-oriented (Majorana) bonds carry an additional factor $\propto \cosh \left(\phi_{\mu}(x)\right)$. Moreover the gauge field introduces an interaction between the two Majorana flavours proportional to $\pm \sinh \left(\phi_{\mu}(x)\right)$, These additional factors introduce a sign problem since each loop can now have an arbitrary sign. However, in the strong coupling limit, the two flavours are bound together. In the present formulation it means that two different Majorana loops lay on top of each other and the resulting double loop describes the world line of the bosonic bound state. It also turns out that all the signs cancel in a non-trivial way and so the bosonisation is realised explicitly. Eventually we end up with a model of non-oriented loops [14] in which all the loop and monomer weights are squared compared to the GN model. Note further that eq.(2.7) no longer applies because the fermionic $\mathrm{BC}$ have no impact on the $\mathrm{BC}$ of the corresponding bosonic bound state - instead the relevant partition function is the one where all topological classes contribute positively, i.e. $Z$.

\section{Simulation algorithm for loops and strings}

A standard procedure to simulate loop gas models as the one described above is to perform local loop updates involving plaquette moves only $[17,18]$. One problem with such an algorithm 

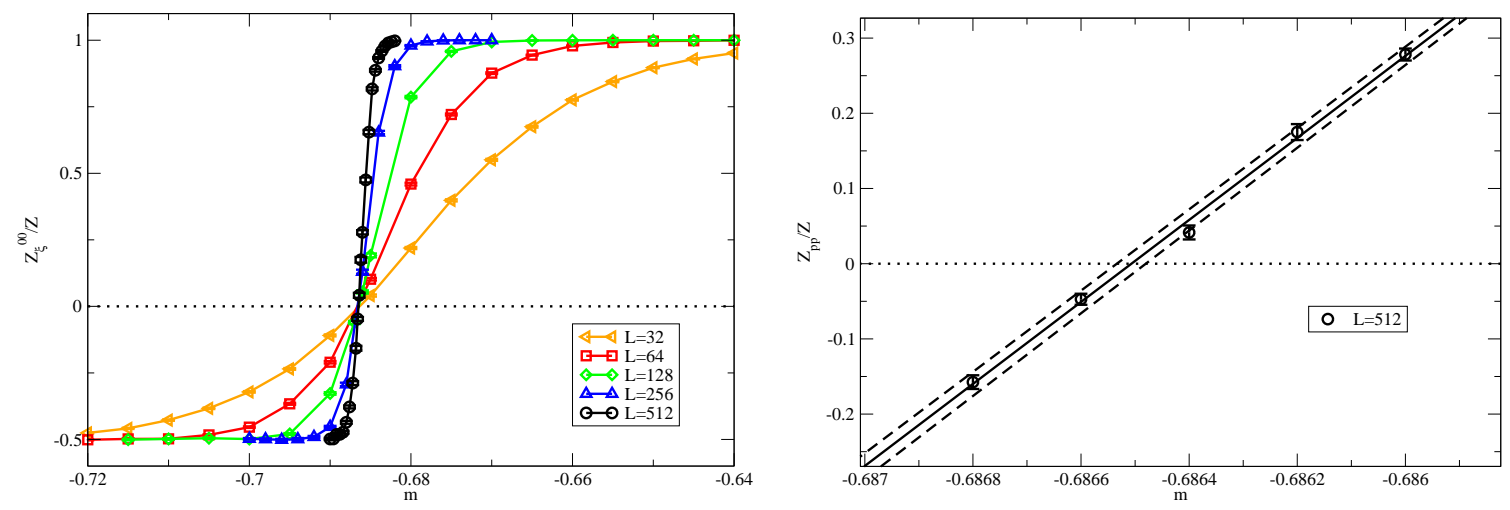

Figure 2: The Schwinger model in the strong coupling limit. Left: Partition function ratio $Z_{\xi}^{00} / Z$ on various lattices. Right: Determination of the critical point $m_{c}=-0.686506(27)$ on the largest lattice with $L=512$.

is that it can not change between the topological classes $\mathscr{L}_{00}, \mathscr{L}_{10}, \mathscr{L}_{01}, \mathscr{L}_{11}$. Moreover, if the correlation length of the system grows large these algorithms become highly inefficient and suffer from CSD. Our proposal [2] (subsequently worked out in [19]) follows the one of Prokof'ev and Svistunov [20] and enlarges the configuration space by open fermionic strings. In the GN model an open string corresponds to the insertion of a Majorana fermion pair $\left\{\xi(x), \xi^{T}(y) \mathscr{C}\right\}$ at position $x$ and $y$ into the path integral, and the open string samples directly the correlation function

$$
G(x, y)=\int \mathscr{D} \xi e^{-S_{\mathrm{GN}}} \xi(x) \xi(y)^{T} \mathscr{C} .
$$

This is the reason why CSD is eliminated: configurations are updated on all length scales up to $O(\zeta)$ where $\zeta$ is the correlation length corresponding to the fermionic two point function. As a consequence the update remains efficient even at a critical point where the correlation length diverges. Contact with the partition functions $Z_{\mathscr{L}_{i j}}$ is made each time the open string closes and this provides the proper normalisation for the expectation value of the 2-pt. function, $\left\langle\xi(x) \xi(y)^{T} \mathscr{C}\right\rangle_{Z}=$ $G(x, y) / Z$, or any other observables. In practice, the ends of the open string are updated with a standard local Metropolis or heat bath procedure [2]. Similar ideas have been around for a long time in various other contexts $[20,21,22]$ - what is new here is the practical application to Wilson fermions and the demonstration that CSD is essentially eliminated.

\section{Absence of critical slowing down}

Before investigating the efficiency of the algorithm, we demonstrate its correctness by comparing simulation results with analytically know expressions. For this purpose we use the $N=1$ Majorana GN model. This model is essentially a free fermion model and can be solved exactly by calculating Pfaffians in momentum space. In the left plot of Figure 1 we show the results for the partition function ratios $Z_{\mathscr{L}_{i j}} / Z$ on a $128^{2}$ lattice from $2 \mathrm{M}$ closed path configurations (symbols) compared to the exact results (dashed lines). The inset shows the combination $Z_{\xi}^{00}=Z_{\mathscr{L}_{00}}-Z_{\mathscr{L}_{10}}-Z_{\mathscr{L}_{01}}-Z_{\mathscr{L}_{11}}$ which has a zero mode at the critical point $m_{c}=0$. The algorithm is indeed able to reproduce the zero mode without problems. In order to investigate the efficiency of the algorithm at the critical point we measure the condensate $\left\langle\xi^{T} \mathscr{C} \xi\right\rangle_{Z_{\xi}}$. The right plot of Figure 1 shows the integrated 

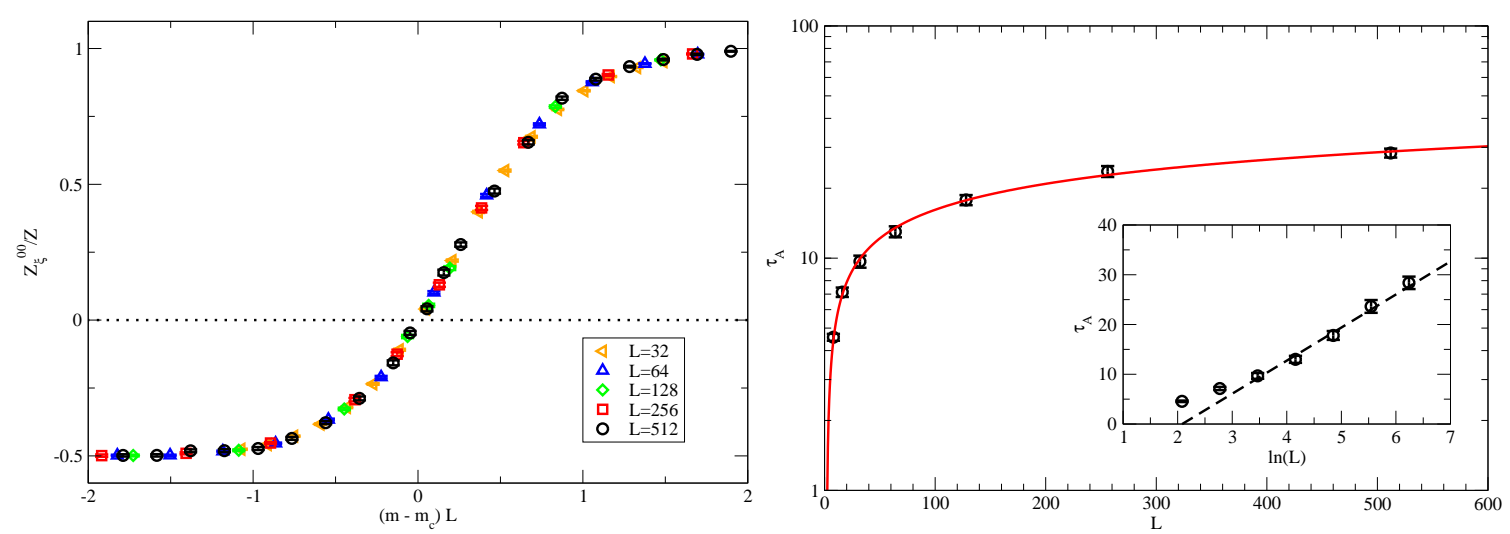

Figure 3: The Schwinger model in the strong coupling limit. Left: Finite size scaling of $Z_{\xi}^{00} / Z$ for a second order phase transition in the universality class of the Ising model. Right: Integrated autocorrelation time of the condensate at the critical point $m_{c}$ fitted by $\tau_{A} \sim L^{z}$ with $z=0.25(2)$. The inset shows a fit to a logarithmic dependence on $L$.

autocorrelation time $\tau_{A}$ of the condensate as a function of the linear system size $L$. The dynamic exponent $z$ relevant for CSD, i.e. $\tau_{A} \sim L^{z}$, turns out to be $z \simeq 0.31(4)$. A dependence logarithmically on $L$ can also be fitted to $L \geq 32$ yielding $-14.2(2.5)+7.1(6) \ln (L)$ with $\chi^{2} / \mathrm{dof}=0.18$.

Next we consider the Schwinger model in the strong coupling limit $g \rightarrow \infty$ as a non-trivial example for strongly interacting fermions. In the left plot of Figure 2 we show the partition function ratio $Z_{\xi}^{00} / Z$ on various lattices up to $L=512$. As in the Majorana GN model we find a zero of the partition function which depends only very little on the extent of the lattice. We can use $Z_{\xi}^{00}\left(m_{c}\right)=0$ as a definition for the critical point $m_{c}$. It can be determined by a linear fit and we obtain $m_{c}=-0.686506(27)$ (cf. right plot in Figure 2) from our simulations on the largest lattice with $L=512$. Further improvement could be achieved by employing standard reweighting techniques as done in [23] where they obtained $m_{c}=-0.6859(4)$. These calculations indicated a second order phase transition in the universality class of the Ising model (with critical exponent $v \simeq 1$ ). Our results in the left plot of Figure 3 now confirm this by demonstrating that the partition function ratios $Z_{\xi}^{00} / Z$ as a function of the rescaled mass $\left(m-m_{c}\right) L^{v}$ with $v=1$ beautifully collapse onto a universal scaling curve. The efficiency of the algorithm and the fact that CSD is essentially absent is demonstrated in the right plot of Fig. 3 where we show the integrated autocorrelation time $\tau_{A}$ of the energy as a function of the linear system size $L$ at the critical point $m=m_{c}$. The functional dependence on $L$ can be well fitted $\left(\chi^{2} /\right.$ dof $\left.=1.28\right)$ by $\tau_{A} \sim L^{z}$ all the way down to our smallest system size $L=8$. We obtain $z=0.25(2)$ which is consistent with just using the largest two system sizes. The autocorrelation time may also depend logarithmically on $L$ and a fit to $L \geq 32$ yields $-13.8(1.9)+6.6(4) \ln (L)$ with $\chi^{2} /$ dof $=1.00$. In any case it is an amazing result that our local Metropolis-type update appears to have a dynamical critical exponent close to zero.

\section{Conclusions}

In conclusion, we have presented a new type of algorithm for Wilson fermions in two dimensions. It relies on sampling directly 2-point correlation functions and essentially eliminates 
critical slowing down. We have successfully tested our algorithm on the Majorana GN model and on the Schwinger model in the strong coupling limit and found remarkably small dynamical critical exponents. The algorithm definitely opens the way to simulate efficiently generic loop models (with positive weights) in arbitrary dimensions, in particular the GN model with any number of flavours, the Thirring model, the Schwinger model and $\mathrm{QED}_{3}$ in the strong coupling limit, as well as fermionic models with Yukawa-type scalar interactions like the $N=1$ and 2 Wess-Zumino models, all with Wilson fermions.

\section{References}

[1] S. Duane, A. D. Kennedy, B. J. Pendleton and D. Roweth, Phys. Lett. B195, 216 (1987).

[2] U. Wenger, Phys. Rev. D80, 071503 (2009), [arXiv:0812.3565].

[3] M. Karowski, R. Schrader and H. J. Thun, Commun. Math. Phys. 97, 5 (1985).

[4] I. Montvay, Phys. Lett. B227, 260 (1989).

[5] C. Gattringer, Int. J. Mod. Phys. A14, 4853 (1999), [cond-mat/9811139].

[6] C. Gattringer, Nucl. Phys. B543, 533 (1999), [hep-lat/9811014].

[7] P. Rossi and U. Wolff, Nucl. Phys. B248, 105 (1984).

[8] F. Karsch and K. H. Mutter, Nucl. Phys. B313, 541 (1989).

[9] D. H. Adams and S. Chandrasekharan, Nucl. Phys. B662, 220 (2003), [hep-lat/0303003].

[10] S. Chandrasekharan and F.-J. Jiang, Phys. Rev. D68, 091501 (2003), [hep-lat/0309025].

[11] S. Chandrasekharan and F.-J. Jiang, Phys. Rev. D74, 014506 (2006), [hep-lat/0602031].

[12] Ph. de Forcrand and M. Fromm, arXiv:0907.1915.

[13] S. Chandrasekharan, PoS LATTICE2008, 003 (2008), [arXiv:0810.2419].

[14] M. Salmhofer, Nucl. Phys. B362, 641 (1991).

[15] U. Wolff, Nucl. Phys. B789, 258 (2008), [arXiv:0707.2872].

[16] I. O. Stamatescu, Phys. Rev. D25, 1130 (1982).

[17] H. Gausterer, C. B. Lang and M. Salmhofer, Nucl. Phys. B388, 275 (1992).

[18] C. Gattringer, V. Hermann and M. Limmer, Phys. Rev. D76, 014503 (2007), [arXiv:0704.2277].

[19] U. Wolff, Nucl. Phys. B814, 549 (2009), [arXiv:0812.0677].

[20] N. Prokof'ev and B. Svistunov, Phys. Rev. Lett. 87, 160601 (2001).

[21] H. G. Evertz, G. Lana and M. Marcu, Phys. Rev. Lett. 70, 875 (1993), [cond-mat/9211006].

[22] O. F. Syljuasen and A. W. Sandvik, Phys. Rev. E66, 046701 (2002).

[23] H. Gausterer and C. B. Lang, Nucl. Phys. B455, 785 (1995), [hep-lat/9506028]. 